\title{
Value-at-Risk da Carteira do lbovespa: uma análise com o uso de modelos de memória longa
}

\section{Value-at-Risk for Ibovespa: an analysis using long memory models}

\author{
Luiz Eduardo Gaio' \\ Tabajara Pimenta Júnior ${ }^{2}$
}

\begin{abstract}
Resumo: O presente estudo propõe uma análise comparativa de dez modelos de volatilidade para o cálculo do Value-at-Risk (VaR) para carteira teórica do Ibovespa, considerando a presença de memória longa na série temporal dos seus retornos diários. Para isso, foram utilizados dados do período de 4 de janeiro de 2000 a 28 de dezembro de 2007. Os resultados mostraram que os modelos que captam o efeito de memória longa na volatilidade condicional dos retornos do Ibovespa, em especial o medido pelo modelo FIGARCH (1,d,1), são os que apresentam melhor desempenho para o cálculo do Value-at-Risk, comparado com alguns modelos tradicionais, como é o caso do Riskmetrics.
\end{abstract}

Palavras-chave: Value-at-Risk. Mercado de capitais. Volatilidade condicional. Ibovespa.

\begin{abstract}
This study proposes a comparative analysis of the use of ten volatility models to calculate the Value-at-Risk (VaR) for Ibovespa considering the presence of long memory in time series for daily returns. Data from January 02, 2000 to January 02, 2008 were used. The results showed that the long memory models, in especial the FICARCH(1,d1) model, are better to calculate the Value-at-Risk if compared to traditional models, such as the RiskMetrics model.
\end{abstract}

Keywords: Value-at-Risk. Stock market. Conditional volatility. Ibovespa.

\section{Introdução}

No segundo semestre de 2008, o mundo viveu um momento conturbado no mercado financeiro internacional. $\mathrm{O}$ que era apenas uma possibilidade de crise tornou-se um fenômeno real. Mais de dez anos depois do desastre do Banco Barings, ocorrido em 1995, grandes corporações financeiras em todo o mundo viram-se em estado de caos com essa crise. Problemas com empréstimos imobiliários para famílias de baixa renda, o chamado mercado subprime, fizeram com que grandes instituições como Merril Lynch, Lehman Brothers, Citibank, UBS, AIG perdessem dezenas de bilhões de dólares em poucos meses.

Grandes desastres financeiros ocorrem, geralmente, pela falta de monitoramento adequado das operações financeiras. Em cenários assim, a gestão de risco ganha força nas instituições financeiras e o estabelecimento de mecanismos de proteção e gestão se torna não só uma importante arma contra as oscilações dos preços dos ativos investidos, mas também ferramenta de análise de investimento. Por conseguinte, o emprego de técnicas que avaliem e mensurem todas as fontes de risco de uma carteira de investimentos possibilita ao gestor manter maior controle sobre onde investir seus recursos, diante de um risco previamente desejado. $\mathrm{O}$ bom gestor de risco não é aquele que elimina todo o risco, mas aquele que possui recursos para gerenciá-lo de forma racional e eficiente.

O grupo J. P. Morgan deu o start inicial no desenvolvimento de ferramentas de gestão de risco. Em 1994 lançou o modelo Riskmetrics para mensuração do risco de mercado de um investimento. Best (1998) conceitua o risco de mercado como o risco de perda resultante de uma variação do valor dos bens transacionáveis. Conforme exposto por Dowd (1998), o Riskmetrics teve origem por iniciativa do ex-presidente do conselho de administração do J. P. Morgan, Dennis Weatherstone, que pediu aos seus funcionários a elaboração um relatório diário contendo um valor referente à exposição sofrida pelo banco ao risco de mercado em todas suas aplicações. Esse relatório deveria ser entregue todos os dias pontualmente às 16 horas e 15 minutos, após o fechamento do mercado. $\mathrm{O}$ valor de risco de mercado pedido por Weatherstone passou a ser chamado, a partir de então, de Value-at-Risk (VaR).

\footnotetext{
${ }^{1}$ Departamento de Administração, Faculdade de Economia, Administração e Contabilidade de Ribeirão Preto - FEA-RP, Universidade de São Paulo - USP, Rua das Palmeiras, 471, CEP 37200-000, Lavras, MG, Brasil , e-mail: lugaio@ yahoo.com.br

${ }^{2}$ Departamento de Administração, Faculdade de Economia, Administração e Contabilidade de Ribeirão Preto - FEA-RP,

Universidade de São Paulo - USP, Av. dos Bandeirantes, 3900, CEP 14049-000, Ribeirão Preto, SP, Brasil
}

Recebido em 30/9/2010 — Aceito em 12/9/2012

Suporte financeiro: Nenhum. 
Depois das iniciativas do J. P. Morgan, pesquisadores de econometria financeira desenvolveram diversos modelos de mensuração e cálculo do risco de mercado que superassem a capacidade preditiva do Riskmetrics. Entre as várias linhas de pesquisa, estudos de risco de mercado adentraram no campo da estatística com a Teoria dos Valores de Extremos, trabalharam com modelos de volatilidade condicional da Economia, abordaram questões de memória longa em séries temporais, provindos das teorias físicas e trabalharam com simulações computacionais e testes de stress, entre outros. No entanto, até o presente momento, não existe uma teoria unificada que consiga mensurar, de forma simples, rápida e com parcimônia, os riscos provindos das oscilações dos mercados.

Dessa forma, a proposta do presente trabalho consiste em responder à seguinte questão: a metodologia de risco proposta pelo RiskMetrics ainda pode ser considerada adequada para a realidade brasileira? ou seja, a incorporação de modelos que capturam o efeito de memória de longo prazo na volatilidade pode prover bom estimador para o cálculo do Value-at-Risk?

Portanto, é proposta uma análise comparativa entre dez métodos de cálculo do Value-at-Risk (VaR) para a carteira teórica do Ibovespa, levando em consideração a presença de longa dependência em seus retornos (memória longa). Dessa forma, pretende-se fazer uma comparação entre modelos de extração da volatilidade para o cálculo do VaR, assim como testar a capacidade de estimação do VaR por meio de um novo modelo proposto por Davidson (2004), o modelo GARCH Hiperbólico (HYGARCH).

\section{Referencial teórico}

\subsection{Gestão de risco}

O risco de mercado está relacionado à possibilidade de perdas oriundas das oscilações do preço de um ativo, ou seja, é a perda causada pelas flutuações do mercado. Esse risco pode, então, ser aplicado a diferentes mercados, como o mercado acionário, mercado de câmbio, mercado de juros, mercado de commodities e outros. Entre os diversos métodos de estimação de risco de mercado, o mais comum usado na gestão do risco para mensurar possíveis perdas no mercado financeiro é o Value-at-Risk (BEST, 1998).

$\mathrm{O}$ VaR é uma das mais importantes ferramentas de mensuração de risco de mercado e, sem dúvida, a mais utilizada pelas instituições e gestores de risco. Estimando a perda máxima esperada, dentro de um período de tempo e um dado nível de confiança, tem-se o conhecido risco de mercado. Por conseguinte, o VaR é um valor que representa, para uma carteira ou ativo, a perda máxima esperada devida aos riscos de mercado para um período de tempo estabelecido e com uma dada probabilidade de ocorrência (JORION, 1997). Por esse método, procura-se prever estatisticamente a variação máxima do valor de mercado de um portfólio num dado intervalo de tempo suficiente para efetuar a liquidação da posição. Jorion (op. cit.) afirma, ainda, que o VaR é um método de mensuração de risco que utiliza padrões de técnicas estatísticas, comumente usadas em outras áreas técnicas.

Dessa forma, uma instituição pode, por exemplo, informar que seu VaR diário é de $\mathrm{R} \$ 10.000$, a um nível de significância de 99\%. Dizer, então, que há apenas uma oportunidade em 100, sob condições normais de mercado, de ocorrer um prejuízo acima de $\mathrm{R} \$ 10.000$. Este único valor resume a exposição da instituição ao risco de mercado, assim como a probabilidade de uma oscilação adversa. Os modelos de análise de risco são, a priori, um conjunto de técnicas que tem como o objetivo gerar este tipo de informação.

Considerando o $W_{0}$ como valor inicial de uma carteira de investimento e $R_{t}$ seu respectivo retorno, o valor esperado da carteira no final do período será:

$$
W_{t}=W_{0}\left(1+R_{t}\right)
$$

Como o interesse é descobrir o valor menor da carteira associado ao nível de confiança dado por (1-c)\%, a taxa de retorno $R_{t}^{*}$ resultante neste menor valor da carteira $W_{t}^{*}$ pode ser dado por:

$$
W_{t}^{*}=W_{0}\left(1+R_{t}^{*}\right)
$$

Examinando o retorno esperado por $\mu$, obtém-se a estimativa do $\mathrm{VaR}$ em relação à média:

$$
\operatorname{VaR}=W_{0}(1+\mu)-W_{0}\left(1+R_{t}^{*}\right)
$$

Simplificando a equação, tem-se:

$$
V a R=-W_{0}\left(R_{t}^{*}-\mu\right)
$$

A ideia principal na obtenção de uma estimativa concisa do VaR reside na capacidade e possibilidade de mensurar com precisão o retorno $R_{t}^{*}$ associada ao valor da carteira $W_{t}^{*}$. Dessa maneira, para essa estimação, é necessário o conhecimento da distribuição de probabilidade dos retornos.

Existem diversos métodos para a estimação do VaR, dependendo das hipóteses formuladas sobre as distribuições de probabilidade dos retornos. Dois desses métodos são bastante conhecidos e utilizados: o primeiro considera as observações históricas como distribuição empírica, é aquele que melhor representa a distribuição de probabilidade dos retornos, e é denominado de VaR não paramétrico (empírico); o segundo, designado de VaR paramétrico, leva em conta a possibilidade de ajuste dos retornos a partir de uma distribuição de probabilidade conhecida, com a sua forma determinada por alguns parâmetros, estimados a partir das observações históricas. $\mathrm{Na}$ 
prática financeira, considera-se que os retornos têm uma distribuição normal. Já no meio acadêmico, outros tipos de distribuição também são ponderados, como é o caso da t-Student, t-Student Assimétrico e GED (Generalized error distruibution)

Para fácil visualização, considerando o quantil Z* da distribuição normal, para o qual a probabilidade c se situa à sua esquerda, pode ser ajustado numa distribuição com média $\mu$ e desvio padrão $\sigma$, para obter o retorno crítico $R_{t}^{*}$ :

$$
R_{t}^{*}=-Z^{*} \sigma+\mu
$$

Substituindo-se o valor por $R_{t}^{*}$ na Expressão 5, obtém-se o Value-at-Risk paramétrico-normal dado por $V a R=W_{0} Z^{*} \sigma$. Pressupondo que os retornos seguem um comportamento independente e identicamente distribuídos (i.i.d.) com distribuição normal, o VaR é estimado apenas pela multiplicação do desvio padrão da carteira, por um fator relativo ao nível de confiança.

A probabilidade adicional deverá ser ajustada pelas caudas, levando em conta que os momentos da distribuição, em específico a variância, oscilam ao longo do tempo. O uso de um modelo de distribuição condicional para extrair essa variação temporal da volatilidade pode ser ajustado na estimação do VaR. Para estimar essa volatilidade, os modelos da família ARCH (Heteroscedasticidade Condicional AutoRegressiva) podem ser usados e, após ser aplicado na Expressão 5, fornecerá o VaR.

\subsection{Modelos de volatilidade}

A modelagem da volatilidade teve origem no trabalho de Engle (1982) e um processo designado por ARCH (AutoRegressive Conditional Heteroskedasticity) expressa a variância condicional como uma defasagem distribuída do quadrado dos retornos passados, ou seja, o retorno de uma série apresenta auto correlação serial, mas a volatilidade (variância condicional) depende de retornos passados por meio de uma função quadrática. Pode ser definido um modelo ARCH (p) por:

$$
\begin{gathered}
R_{t}=\sqrt{\sigma_{t}^{2}} \varepsilon_{t} \\
\sigma_{t}^{2}=\alpha_{0}+\sum_{i=1}^{p} \alpha_{i} R_{t-i}^{2}
\end{gathered}
$$

em que $R_{t}^{*}$ é o retorno, $\varepsilon_{\mathrm{t}}$ é uma sequência de variáveis aleatórias independentes e identicamente distribuídas (i.i.d.) com média zero e variância um e $\alpha_{0}>0, \alpha_{i} \geq 0$ e $i>0$.

Vale ressaltar que Morettin e Toloi (2004) afirmam que, na prática, deve-se supor usualmente $\varepsilon_{\mathrm{t}} \sim \mathrm{N}(0,1)$ ou $\varepsilon_{\mathrm{t}} \sim t v$ ( $t$ de Student com $v$ graus de liberdade).

Conforme Mol (2003), esse modelo de variância condicional possui algumas propriedades desejáveis.
Em primeiro lugar, por meio da técnica de decomposição de erros de predição, é possível construir a função de verossimilhança, tornando possível a estimação dos parâmetros pelo método de máxima verossimilhança. Esta propriedade é importante porque esses estimadores possuem distribuições conhecidas que viabilizam a execução de testes de hipóteses diversos. Além disso, é possível provar que este modelo implica uma distribuição não condicional com "caudas pesadas" para os retornos.

De acordo com Goulart et al. (2005), o modelo original, elaborado por Engle (1982), obteve muitos debates e diversos aperfeiçoamentos ao longo dos anos. O primeiro, e mais utilizado no meio acadêmico, foi relacionado aos estudos de Bollerslev (1986) e propõe que a volatilidade condicionada fosse função não apenas dos quadrados dos erros passados $\left(R_{t}^{2}\right)$, como também dos seus próprios valores $\left(\sigma_{t-1}^{2}\right)$, e os modelos assim construídos passam a ser denominados Generalized ARCH (GARCH). Um modelo GARCH $(p, q)$ pode ser expresso como:

$$
\sigma_{t}^{2}=\alpha_{0}+\sum_{i=1}^{p} \alpha_{i} R_{t-i}^{2}+\sum_{j=1}^{q} \beta_{j} \sigma_{t-j}^{2}+v_{t}
$$

em que $\alpha_{0}$ é a constante, $v_{t}$ é um ruído branco $[\mathrm{N} \sim(0,1)]$

A fim de que a variância condicional não assuma valores negativos e também haja a estacionariedade do processo, tem-se que $\alpha_{0}>0 ; \alpha_{i} \geq 0$, para $\mathrm{i}=1$, $\ldots, \mathrm{p} ; \beta_{j} \geq 0$, para $\mathrm{j}=1, \ldots, \mathrm{q} ;$ e $\sum_{i=1}^{p} \alpha_{i}+\sum_{j=1}^{q} \beta_{j}<1$.

Uma vantagem do modelo GARCH comparado ao modelo ARCH dá-se pelo fato de o modelo GARCH ser parcimonioso, ou seja, o modelo ARCH geralmente requer mais parâmetros de especificação do que o modelo GARCH.

Baseados nos princípios dos modelos acima descritos, vários pesquisadores observaram a existência de deficiências para determinadas séries econômicas. Silva (2003) postulou que, mesmo o processo GARCH sendo flexível e várias extensões deste modelo tenham sido propostas, de forma a incluir características particulares verificadas em determinadas séries econômicas, como assimetrias e mudanças de regime, os processos GARCH não captam adequadamente algumas regularidades encontradas concernentes à persistência, ou memória longa, na volatilidade do retorno de algumas séries econômicas.

Por conseguinte, com o objetivo de introduzir a teoria dos modelos de memória longa aplicados à volatilidade dos retornos, serão expostos primeiramente os principais conceitos que embasam este artigo, relativos aos processos fracionalmente integrados para a média para, então, serem apresentadas a metodologia e a análise e discussão dos dados.

Morettin e Toloi (2004) descreveram que o processo ARMA $(p, q)$ é referenciado como um processo de "memória curta", uma vez que sua função 
de autocorrelação tende a exibir um decaimento exponencial, ou seja, decresce rapidamente a zero. Para eles, um processo de memória longa é um processo estacionário em que a função de autocorrelação decresce hiperbolicamente (suavemente) para zero. Outra importante característica de séries com memória longa apresentada por esses autores são as autocorrelações da série original, as quais indicam não estacionaridade, ao passo que a série diferençada pode ser "superdiferençada".

Buscando criar um modelo que conseguisse captar a presença de memória longa, Hosking (1981) e Granger (1980) incorporaram ao processo ARMA $(\mathrm{p}, \mathrm{q})$ o operador de diferenciação fracionária $(1-\mathrm{B})^{d}$, sendo $0 \leq \mathrm{d} \leq 1$, em que $\mathrm{B}$ representa o operador de defasagem. Assim, obtiveram um processo ARFIMA $(\mathrm{p}, \mathrm{d}, \mathrm{q})$ (Autorregressivo Fracionalmente Integrado de Médias Móveis), com especificação dada por,

$$
\phi(B)(1-B)^{d} X_{t}=\theta(B) \varepsilon_{t}
$$

em que $\left\{\varepsilon_{\mathrm{t}}\right\}$ é um ruído branco e $\phi(\mathrm{B})$ e $\theta(\mathrm{B})$, são polinômios em $\mathrm{B}$ de graus $\mathrm{p}$ e q, respectivamente. Morettin e Toloi (2004) descreveram o operador de diferenciação fracionária como,

$$
\begin{aligned}
& (1-B)^{d}=\sum_{k=0}^{\infty}\left(\begin{array}{l}
d \\
k
\end{array}\right)(-B)^{k} \\
& =1-d B+\frac{1}{2 !} d(d-1) B^{2} \\
& -\frac{1}{3 !} d(d-1)(d-2) B^{3}+\ldots
\end{aligned}
$$

Silva (2003) afirmou que um modelo ARFIMA impõe uma dicotomia entre a dinâmica de curto e longo prazo, modelando o comportamento de curto prazo por meio da estrutura ARMA tradicional, enquanto a dinâmica de longo prazo é modelada pelo parâmetro de diferenciação fracionária $\mathrm{d}$.

A partir disso, para imitar o comportamento do correlograma observado para a volatilidade das séries, Baillie, Bollerslev e Mikkelsen (1996) introduziram o modelo GARCH fracionalmente integrado (FIGARCH) que busca captar a memória longa na variância condicional. A ideia desses autores surgiu do modelo IGARCH (p,q) desenvolvido por Engle e Bollerslev (1986).

O modelo IGARCH $(\mathrm{p}, 1)$ segue a seguinte expressão:

$$
\phi(B)(1-B) X_{t}^{2}=\alpha_{0}+[1-\beta(B)]\left(X_{t}^{2}-\sigma_{t}^{2}\right)(11)
$$

em que $\phi(B)=[1-\alpha(B)-\beta(B)](1-B)^{-1}, \quad$ o processo FIGARCH (p,d,q) pode ser obtido substituindo o operador de primeira diferença $(1-B)$ pelo operador de diferença fracionária $(1-B)^{d}$.
Assim, a variância condicional do FIGARCH (p,d,q) é dada por:

$$
\begin{aligned}
& \sigma_{t}^{2}=\underbrace{\alpha_{0}[1-\beta(B)]^{-1}}_{\alpha_{0} *} \\
& +\underbrace{\left\{1-[1-\beta(B)]^{-1} \phi(B)(1-B)^{d}\right\}}_{\lambda(B)} X_{t}^{2}
\end{aligned}
$$

ou $\sigma_{t}^{2}=\alpha_{0} *+\sum_{i=1}^{\infty} \lambda_{i} B^{i} X_{t}^{2}=\alpha_{0} *+\lambda(B) X_{t}^{2}$, com $0 \leq d \leq 1$.

Segundo Baillie, Bollerslev e Mikkelsen (1996), a amplitude (S) do processo FIGARCH é $\mathrm{s}=\lambda(1)=1$. Assim, o termo $\alpha_{0}>0$ teria a mesma interpretação válida para o modelo IGARCH. Consequentemente, o segundo momento da distribuição incondicional de $X_{t}$ seria infinito e o processo FIGARCH não fracamente estacionário.

Em contrapartida, Davidson (2004) demonstrou como a restrição arbitrária $(S=\lambda(1)=1)$ explica o estranho comportamento da memória do processo FIGARCH. Ele acrescenta que a duração da memória aumenta à medida que $\mathrm{d}$ tende a zero. Este é um contraste à interpretação convencional, a qual sugere que a duração da memória aumenta quando d cresce.

Para Davidson (2004), que desenvolveu o processo GARCH hyperbólico (HYGARCH), o qual é considerado como a generalização do modelo FIGARCH, o modelo HYGARCH introduz outro parâmetro $\alpha$ no lag polinomial. Portanto, o modelo HYGARCH $(1, \mathrm{~d}, 1)$ pode ser expresso como

$$
\sigma_{t}^{2}=\frac{\alpha_{0}}{1-\beta}+\left(1-\frac{(1-\phi B)\left[1+\alpha\left((1-B)^{d}-1\right)\right]}{(1-\beta B)}\right) X_{t}^{2}(13)
$$

desde que $\mathrm{d}>0$, a amplitude do modelo HYGARCH $(1, \mathrm{~d}, 1)$ é $S=1-\frac{\phi}{\beta}(1-\alpha)$.

O modelo HYGARCH, portanto, não impõe a restrição da amplitude ser igual a 1.

\subsection{Metodologia RiskMetrics}

A abordagem seguida na metodologia Riskmetrics implementa, precisamente, a volatilidade condicional na Expressão 8, por meio do modelo GARCH.

Segundo a metodologia Riskmetrics, a variância condicional ótima é estimada por um modelo GARCH $(1,1)$ com constante $\alpha_{0}$ igual a zero e a soma dos parâmetros $\alpha$ e $\beta$ igual à unidade. Dessa forma, obtêm-se o processo formalmente conhecido por GARCH integrado (IGARCH): 


$$
\sigma_{t}^{2}=\lambda \sigma_{t-1}^{2}+(1-\lambda) R_{t-1}^{2}
$$

ou

$$
\sigma_{t}^{2}=\lambda^{t} \sigma_{0}^{2}+(1-\lambda) \sum_{K=0}^{t-1} \lambda^{k} R_{t-k}^{2}
$$

em que $\sigma_{0}^{2}$ é um dado nível da variância no momento inicial. A taxa de declínio das ponderações exponenciais depende do Fator de Decaimento- $\lambda$, o qual exprime a persistência com que os efeitos de um choque se fazem sentir no futuro (MORGAN, 1996).

Fatores de Decaimento- $\lambda$ de 0.94 para retornos diários e 0.97 para retornos mensais são sugeridos por Morgan (1996). Segundo ele, o fato de apenas ser necessário utilizar um parâmetro, $\lambda$, facilita a estimação da volatilidade condicional e proporciona robustez contra o erro de estimação, apesar da parcimoniosidade do modelo.

\subsection{Evidências empíricas de memória longa para o cálculo do $\mathrm{VaR}$}

O Value-at-Risk é um dos mais utilizados métodos de mensuração e análise de risco de mercado e tem, pois, sido utilizado por diversos gestores de risco em instituições financeiras, bolsa de valores, clearing house e administradores de carteira. Desde o lançamento oficial do pacote Riskmetrics em 1994, pelo grupo de gestão de risco do J.P. Morgan, este modelo tem sido referência na mensuração de risco de mercado.

Depois do lançamento do Riskmetrics, diversas pesquisas vêm sendo desenvolvidas para se testar a real eficiência desta metodologia na mensuração do risco de mercado, em específico o cálculo do Value-at-Risk. Entre os diversos trabalhos desenvolvidos, a pesquisa realizada por Giot e Laurent (2003a) tem sido uma referência na área. Esses autores testaram o modelo de volatilidade condicional APARCH, utilizando a distribuição $t$-Student assimétrica, como ferramenta de cálculo do VaR diário dos índices Dow Jones, FTSE, Nasdaq e Nikkei. Como resultados, eles encontraram que o modelo APARCH $t$-Student Assimétrico obteve um desempenho muito superior ao modelo Riskmetrics, tanto para posições curtas como longas. Dessa forma, Giot e Laurent (2003a) abriram uma nova lacuna para discussão sobre o assunto de gestão de risco.

Em continuidade à pesquisa, Giot e Laurent (2003b) sugeriram o mesmo modelo de estimação de risco testado anteriormente, mas aplicando no mercado futuro de commodities norte-americanas, como alumínio, cobre, cacau e petróleo. Novamente, os resultados foram semelhantes aos anteriores, mostrando a superioridade dos modelos APARCH $t$-Student Assimétrico como ferramenta de estimação do risco.
Já em estudos posteriores, Giot e Laurent (2004) testaram a capacidade preditiva do modelo APARCH t-Student Assimétrico utilizando como proxy a volatilidade realizada dos índices CAC40, SP500, YEN-USD e DEM-USD. Os resultados não foram diferentes dos esperados e o modelo APARCH novamente teve melhor desempenho para estimar o VaR diário.

Huang e Lin (2004) fizeram um estudo semelhante aos realizados por Giot e Laurent (2003a), visando testar o modelo de volatilidade condicional APARCH comparados com o pacote do J. P. Morgan, Riskmetrics. Utilizaram como distribuição para os retornos a Normal e a $t$-Student. A amostra de dados utilizada foram os índices futuros de Taiwan TAIFEX e SGX-DT e os resultados evidenciaram a supremacia dos modelos de volatilidade, em específico o APARCH $t$-Student, comparados ao modelo Riskmetrics.

No ano de 2005, não foram evidenciados estudos comparativos das metodologias de estimação da volatilidade aplicados ao cálculo do Value-at-Risk. Os estudos neste período centraram em testar modelos da classe ARCH de forma mais empírica, com poucas aplicações ao mercado financeiro de gestão do risco.

Partindo do pressuposto de que os retornos de um determinado mercado podem apresentar uma longa persistência, So e Yu (2006) fizeram uma pesquisa ampla, testando três modelos da classe ARCH - GARCH, IGARCH e FIGARCH - assumindo duas diferentes distribuições dos retornos, Normal e $t$-Student, comparados com o modelo Riskmetrics tradicional. Seu diferencial foi a utilização do modelo FIGARCH, que tem a capacidade de modelar o comportamento de longa dependência da volatilidade dos retornos. Para a realização dos estudos, foram utilizados 12 índices do mercado financeiro global, AOI, FTSE100, HSI, JSX, KLSE, KOSPI, Nasdaq, Nikkei, SET, SP500, STII e WEIGHT. Como resultado, evidenciou-se que, para todos os casos, o modelo FIGARCH obteve melhores resultados que os demais, superando também o modelo Riskmetrics.

Tang e Shieh (2006) investigaram a evidência de memória longa nos preços de fechamento de três índices futuros: S\&P500, Nasdaq 100 e Dow Jones. Para tanto, foram utilizados os modelos de volatilidade condicional FIGARCH $(1, \mathrm{~d}, 1)$ e HYGARCH $(1, \mathrm{~d}, 1)$, com três diferentes distribuições, Normal, $t$-Student $e$ t-Student Assimétrica. Os resultados mostraram que o modelo HYGARCH com distribuição $t$-Student Assimétrica foi o que apresentou melhor performance de estimação do VaR para os três diferentes índices, reforçando a hipótese de memória longa na volatilidade dos retornos internacionais.

Testando a eficácia dos modelos de memória longa comparados com os tradicionais da família ARCH, em específico, Wu e Shieh (2007) testaram os modelos GARCH $(1,1)$ e FIGARCH $(1, d, 1)$, assumindo 
três diferentes distribuições dos retornos, Normal, $t$-Student e $t$-Student Assimétrica. A pesquisa foi realizada nos retornos futuros do T-Bond diário. Os resultados empíricos mostraram que os modelos com distribuição $t$-Student Assimétrica possuem melhor qualidade de ajuste. O modelo FIGARCH $(1, \mathrm{~d}, 1)$ foi um dos modelos com melhor desempenho para o cálculo do VaR.

Outro estudo empírico, com a finalidade de descobrir a performance de previsão do VaR aplicado ao índice da Bolsa de Valores de Madrid, foi o estudo realizado por Níguez (2008), em que os modelos de volatilidade condicional, GARCH, AGARCH, APARCH, EWMA, FIGARCH e FIAPARCH foram testados, assumindo distribuições normal e $t$-Student. Os resultados desse estudo evidenciaram que o modelo FIAPARCH com distribuição $t$-Student foi o que melhor estimou os valores do VaR tanto para posições curtas quanto para longas, de acordo com o teste de Kupiec (1995).

Em âmbito nacional, poucos foram os estudos que analisam o comportamento da volatilidade para o cálculo do Value-at-Risk. Entre os principais, pode-se citar Mota e Fernandes (2004), Moreira e Lemgruber (2004), Galdi e Pereira (2007) e Maciel (2010).

Mota e Fernandes (2004) realizaram um estudo que investiga o desempenho de estimadores de volatilidade aplicados ao índice Bovespa, tendo como referência a volatilidade realizada. Foram comparados modelos da classe ARCH com alguns alternativos, baseados em cotações de abertura, fechamento, máximo e mínimo. Os resultados evidenciaram que os modelos alternativos são precisos para extração da volatilidade e cálculo do Value-at-Risk, assim como os autorregressivos.

Moreira e Lemgruber (2004) investigaram o uso de dados de alta frequência na estimação da volatilidade para o cálculo do risco de mercado. Em sua metodologia, utilizaram-se diversos métodos determinísticos de filtragem de sazonalidade, para correção das anomalias presentes no comportamento do Ibovespa. Para ajuste da volatilidade, utilizaram-se os modelos ARCH e GARCH. A base de dados refere-se ao índice Bovespa no mercado intradiário, período de 15 minutos entre os anos de 1998 e 2000. Os resultados evidenciaram que o uso de modelos de volatilidade sem a prévia correção sazonal gera fortes distorções da estimação do risco de mercado. Portanto, a utilização de métodos de filtragem sazonal é indispensável para o ajuste do risco.

Galdi e Pereira (2007) fizeram um estudo comparativo de estimadores de volatilidade para o cálculo do valor em risco, aplicados ao mercado nacional. Os modelos considerados para ajustar a volatilidade foram Suavização exponencial - EWMA, volatilidade condicional - GARCH e volatilidade estocástica. Para a avaliação, foram utilizadas cotações diárias dos preços das ações da Petrobras no período de 1995 a 2006. Os resultados apresentados demonstram que os modelos com suavização exponencial sofrem menos distorções do que os condicionais e estocástica.

Maciel (2010) fez uma análise comparativa de estimadores do Valor em Risco (VaR) de longo prazo utilizando modelos da classe $\mathrm{ARCH}$ e redes neurais artificiais, aplicados ao mercado brasileiro de capitais. A metodologia utilizada refere-se aos modelos GARCH $(1,1)$ e Rede Neuronal multicamadas, nos retornos das empresas Petrobrás (PETR4), Vale do Rio Doce (VALE5), Grupo Gerdau (GGBR4), Banco Itaú Unibanco (ITAU4) e BM \& FBOVESPA (BMEF3), no período de 2000 a 2006. Os resultados mostraram que o modelo Riskmetrics superestima o valor em risco $(\mathrm{VaR})$, quando comparado aos demais.

\section{Metodologia}

\subsection{Dados da pesquisa}

Foram coletados os valores de fechamento diários do índice de ações da Bolsa de Valores de São Paulo (Ibovespa) no período de 2 de janeiro de 2000 a 28 de dezembro de 2007 num total de 1976 observações, a partir da base de dados do ECONOMÁTICA ${ }^{\circledR}$. O ano de 2008 foi excluído da amostra devido às grandes oscilações sofridas no mercado, podendo mesclar a estimação da volatilidade e teste de capacidade preditiva.

Para o estudo de análise e mensuração do risco, foi utilizada uma série de retorno do índice Bovespa, conforme expressão;

$$
R_{t}=\ln \left(\frac{I B V_{t}}{I B V_{t-1}}\right)=\ln \left(I B V_{t}\right)-\ln \left(I B V_{t-1}\right)(16)
$$

em que $I B V$ representa a série de fechamento das cotações do índice Bovespa e $R_{t}$ a série do retorno do Ibovespa.

A utilização da série de retorno como entrada para os testes estatísticos é bastante utilizada no meio acadêmico por se tratar de dados já estacionários com média zero e variância $\sigma$. Assim, a série de retornos substituiu a série original de cotações do índice.

A Figura 1 expressa a evolução das cotações do Ibovespa entre 4 de janeiro de 2000 a 28 de dezembro de 2007.

Na Figura 2, são apresentados os retornos logarítmicos calculados dos índice BOVESPA no período de 4 janeiro de 2000 a 28 de dezembro de 2007.

Nas Figuras 1 e 2, é perceptível que o Ibovespa apresenta um comportamento volátil no decorrer do tempo e seus retornos possuem um comportamento semelhante a uma normal, com média 0 e variância $\sigma^{2}$. Porém tais fatos não podem ser afirmados sem a 


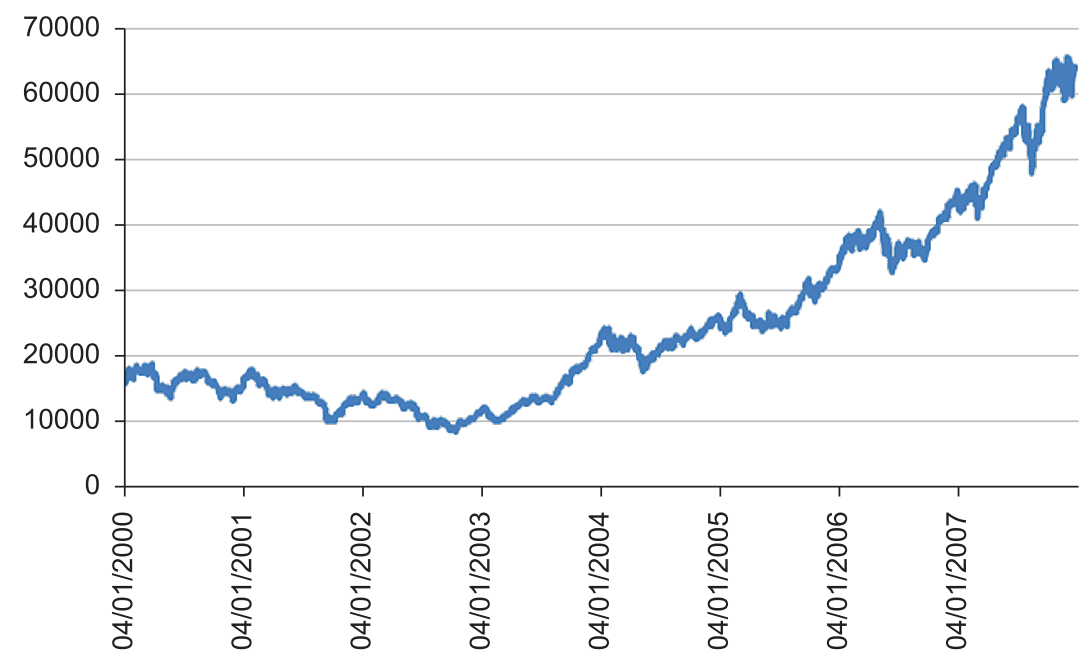

Figura 1. Evolução do Ibovespa.

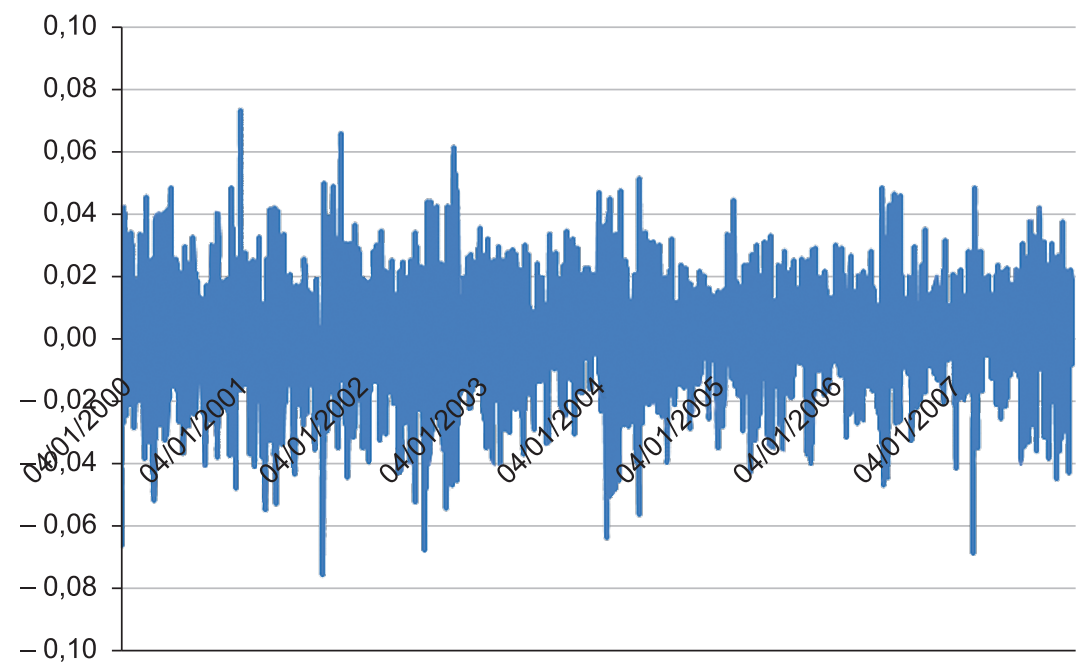

Figura 2. Evolução dos retornos Ibovespa.

realização de testes que comprovem o comportamento dos retornos.

Os dados foram tratados pelo programa Excel, excluindo sábados, domingos, feriados e dias sem negociação e, para a utilização de métodos estatísticos, foi utilizado o pacote estatístico G@RCH 2,3 do software $O x^{\circledR}$ sugerido por Laurent e Peters (2002). Já os detalhes de operacionalização do sistema foram obtidos em Doornik (2001).

\subsection{Análise dos dados}

A investigação empírica iniciou-se com a análise das estatísticas descritivas da série e aplicação dos testes de normalidade, estacionariedade. Para testar a normalidade da distribuição da série de retornos, foi aplicado o teste de Jarque-Bera (BERA; JARQUE, 1981), acompanhado de seu p-valor, ou seja, a probabilidade de não rejeitar a hipótese nula de que a série segue um padrão normal. O nível de confiança definido foi o de $95 \%$ e o teste de estacionariedade foi feito a partir dos testes de raiz unitárias Dickey e Fuller Aumentado (ADF) (DICKEY; FULLER, 1979), Phillips e Perron (PP) (PHILLIPS; PERRON, 1979) e KPSS (KWIATKOWSKI et al., 1992), também acompanhado de seu p-valor, ou seja, a probabilidade da série apresentar uma raiz unitária. O intervalo de confiança adotado foi, assim como no teste de normalidade, de $95 \%$.

O passo seguinte do estudo foi a realização do teste de heteroscedasticidade ARCH-LM, proposto por Engle (1982), com o intuito de se verificar a presença do efeito de heteroscedasticidade condicional na série de retorno no Ibovespa. Essa presença é fator determinante para a aplicação dos modelos da classe ARCH. 
Já o terceiro passo consistiu na modelagem da variância condicional dos retornos do Ibovespa a partir do modelo Riskmetrics, modelo GARCH tradicional e modelos de memória longa FIGARCH e HYGARCH. É interessante ressaltar que, para o ajustamento e estimação dos modelos acima descritos, com exceção do Riskmetrics, foram utilizados três tipos de distribuições para os resíduos: distribuição normal (Gaussiana), t-Student, e t-Student Assimétrica. Dessa forma, têm-se três ajustamentos para o modelo GARCH, ajustamento I (distribuição Normal), ajustamento II (t-Student) e ajustamento III (t-Student Assimétrico), os quais valem para todos os modelos, totalizando dez ajustamentos.

O próximo passo estimou os riscos de mercado do Ibovespa. Para isso, foi calculado o VaR para os dados dentro da amostra (In-Sample) e estimativas fora da amostra (Out-of-Sample), em posições curtas, quantis de $0.95,0.975$ e 0.99 , e posições longas, quantis de $0.05,0.025$ e 0.01 .

Por fim, o quinto e último passo do estudo foi o cálculo da estatística de Kupiec, para cada estimativa de Value-at-Risk, com o intento de se verificar a acurácia do VaR calculado.

\subsection{O Teste de Kupiec}

Para se testar o desempenho de um estimador do VaR, Kupiec (1995) desenvolveu um teste baseado na quantidade de falhas na previsão do risco de mercado, comparado com o intervalo de confiança estabelecido a priori.

Considerando $\alpha$ o intervalo de confiança estabelecido no cálculo do VaR, o teste LR Kupiec visa testar a hipótese nula de que $H_{0}: f=\alpha$ contra a hipótese alternativa $H_{0}: f \neq \alpha$ em que $f$ é a taxa de falha. Se o modelo estiver corretamente especificado, $f$ deve ser igual a $\alpha$.

A taxa de falha pode ser estimada conforme expressão:

$$
f=\frac{x}{N}
$$

em que $x$ é o número de retornos que excedem o VaR calculado e $N$ é o número total da amostra.

De acordo com Kupiec (1995), $x$ segue uma distribuição $x \sim$ Binominal $(\mathrm{N}, f)$, cuja probabilidade de $x$ na amostra $N$ é dado por $P(x, f, N)=C_{x}^{N}(1-f)^{N-x} f^{x}$.

Esse autor propôs, ainda, o teste baseado na razão de verossimilhança, que pode ser empregado à estimativa de uma amostra pontual estatisticamente consistente com o modelo VaR.

A estatística de teste é dada por:

$$
\begin{aligned}
& L R=-2 \ln \left[(1-\alpha)^{N=x} \alpha^{x}\right] \\
& +2 \ln \left[(1-f)^{N-x} f^{x}\right] \sim \chi^{2}(1)
\end{aligned}
$$

O teste segue uma distribuição de quiquadrado com 1 grau de liberdade.

\section{Resultados e discussões}

A Tabela 1 apresenta a estatística descritiva e o resultado dos testes de raiz unitária ADF, PP e KPSS, aplicados à série de retorno do índice Bovespa.

Conforme os valores apresentados na Tabela 1, pode-se perceber que a série de retorno do Ibovespa apresenta um comportamento não normal, percebido a partir do teste de normalidade de Jarque-Bera,

Tabela 1. Estatística Descritiva da série de retornos do Ibovespa.

\begin{tabular}{lc}
\hline Período & $1 / 1 / 2000$ a $11 / 9 / 2007$ \\
Média & 0,0006007 \\
Máximo & 0,073353 \\
Mínimo & $-0,096342$ \\
Desvio Padrão Incondicional & 0,018193 \\
Assimetria & $-0,247106$ \\
Curtose & 3,98531 \\
& \\
Jarque-Bera & $96,64966^{*}$ \\
p-valor & 0,00 \\
Observações & 1909 \\
Teste ADF & $-42,46448^{*}$ \\
Teste PP & $-42,47175^{*}$ \\
Teste KPSS & $0,37831^{*}$ \\
\hline
\end{tabular}

Os valores críticos do teste de ADF e PP são-3,4358 ao nível de 1\% de significância. *Significativo a 1\% de significância. 
rejeitado ao nível de $1 \%$ da hipótese de normalidade, considerado pelo $\mathrm{p}$-valor igual a zero. $\mathrm{O}$ excesso de curtose, com valor acima de 3 , é um dos principais fatores que pode ter gerado a rejeição da hipótese de normalidade. $\mathrm{O}$ valor 3,98 da curtose indica que a série de retornos apresenta um comportamento leptocúrtico, com caldas pesadas.

O comportamento leptocúrtico e assimétrico é bastante comum em séries financeiras, principalmente em retornos de mercados, o que vai de acordo com o previsto pela teoria financeira.

Os resultados empíricos do teste de raiz unitária apresentados pela Tabela 1 apontam para uma estacionaridade da série de retorno nos três testes, a hipótese nula de raiz unitária pode ser rejeitada ao nível de $1 \%$. Realizaram-se diversos testes considerando a presença do intercepto e, também, o efeito de possíveis tendências. $\mathrm{O}$ critério de escolha do melhor modelo foi aquele que apresentou menores valores de AIC (Akaike Information Criterion) e BIC (Bayesian Information Criterion).

As Tabelas 2, 3, e 4 apresentam os valores dos parâmetros estimados dos modelos GARCH $(1,1)$, FIGARCH $(1, d, 1)$, HYGARCH $(1, d, 1)$ para distribuições normais, t-Student e t-Student Assimétrica. Os estimadores de máxima verossimilhança para os parâmetros dos modelos de memória longa utilizados são calculados com o método BBM descrito por Baillie, Bollerslev e Mikkelsen (1996).

De forma geral, observa-se que os modelos testados obtiveram um bom ajuste à volatilidade dos retornos, por apresentarem parâmetros significativos em quase todos os casos, ao nível de $1 \%$ de significância. Ressalta-se, também, que o efeito heteroscedástico pôde ser eliminado em todos os modelos, uma vez que a hipótese nula de homocesticidade é rejeitada

Tabela 2. Parâmetros estimados modelo GARCH $(1,1)$.

\begin{tabular}{lccc}
\hline & Normal & $\boldsymbol{t}$-Student & $\boldsymbol{t}$-Student assimétrica \\
\hline Constante & $0,107756(0,0052)$ & $0,077268(0,0210)$ & $0,074552(0,0171)$ \\
ARCH (Alpha1) & $0,057088(0,0000)$ & $0,053954(0,0000)$ & $0,053449(0,0000)$ \\
GARCH (Beta1) & $0,910039(0,0000)$ & $0,922804(0,0000)$ & $0,923736(0,0000)$ \\
Student & & $13,63212(0,0001)$ & \\
Asymmetry & & & $-0,122917(0,0006)$ \\
Tail & & & $15,980301(0,0006)$ \\
& & & \\
Log-verossimilhança & 4992,035 & 5004,453 & 5010,563 \\
AIC & $-5,223012$ & $-5,233548$ & $-5,240512$ \\
Q $^{2}(10)$ & 7,08292 & 6,83614 & 6,80562 \\
ARCH $(10)$ & 1,0986 & 1,1502 & 1,1454 \\
\hline
\end{tabular}

Os valores entre parênteses correspondem ao p-valor de interpretação direta. Log-verossimilhança é o valor máximo da função de verossimilhança logarítmica. Q²(10) corresponde a Q-estatística de Ljung-Box para o décimo lag do erro quadrático. ARCH (10) refere-se ao Teste ARCH-LM para o lag 10.

Tabela 3. Parâmetros estimados modelo FIGARCH $(1,1)$.

\begin{tabular}{lccc}
\hline & Normal & $\boldsymbol{t}$-Student & $\boldsymbol{t}$-Student assimétrica \\
\hline Constante & $0,444209(0,0054)$ & $0,297214(0,0197)$ & $0,287174(0,01900)$ \\
D & $0,249460(0,000)$ & $0,295924(0,0000)$ & $0,298079(0,0000)$ \\
ARCH (Alpha1) & $0,025720(0,8106)$ & $0,070276(0,4613)$ & $0,063766(0,09325)$ \\
GARCH (Beta1) & $0,270485(0,0274)$ & $0,036214(0,0024)$ & $0,359071(0,0022)$ \\
Student & & $13,61072(0,0001)$ & \\
Asymmetry & & & $-0,126582(0,0004)$ \\
Tail & & & $15,915173(0,0004)$ \\
& & & \\
Log-verossimilhança & 4993,896 & 5010,372 & 5016,786 \\
AIC & $-5,227759$ & $-5,23869$ & $-5,245914$ \\
$Q^{2}(10)$ & 7,61716 & 7,38334 & 7,37207 \\
ARCH (10) & 0,30877 & 0,3782 & 0,37369 \\
\hline
\end{tabular}

Os valores entre parênteses correspondem ao p-valor de interpretação direta, Log-verossimilhança é o valor máximo da função de verossimilhança logarítmica, $\mathrm{Q}^{2}(10)$ corresponde a Q-estatística de Ljung-Box para o décimo lag do erro quadrático, ARCH (10) refere-se ao Teste ARCH-LM para o lag 10, 
Tabela 4. Parâmetros estimados modelo HYGARCH $(1,1)$.

\begin{tabular}{lcrc}
\hline & Normal & $\boldsymbol{t}$-Student & t-Student assimétrica \\
\hline Constante & $0,001017(0,0083)$ & $0,284666(0,2647)$ & $0,309251(0,1862)$ \\
D & $0,488631(0,0436)$ & $0,287839(0,0563)$ & $0,313066(0,0372)$ \\
ARCH (Alpha1) & $0,028773(0,7844)$ & $0,069535(0,4764)$ & $0,06483(0,4782)$ \\
GARCH (Beta1) & $0,296116(0,0555)$ & $0,35684(0,0184)$ & $0,368773(0,0119)$ \\
Log alpha (HY) & $-0,06582(0,7442)$ & $0,012318(0,9553)$ & $-0,020771(0,9099)$ \\
Student & & $13,59415(0,0001)$ & \\
Asymmetry & & & $-0,126689(0,0004)$ \\
Tail & & & $15,960418(0,0005)$ \\
& & & 5016,792 \\
Log-verossimilhança & 4997,422 & 5010,373 & $-5,244876$ \\
AIC & $-5,226766$ & $-5,237643$ & 7,37321 \\
$Q^{2}(10)$ & 7,62369 & 7,38313 & 0,3818 \\
ARCH (10) & 0,31047 & 0,38067 & \\
\hline
\end{tabular}

Os valores entre parênteses correspondem ao p-valor de interpretação direta, Log-verossimilhança é o valor máximo da função de verossimilhança logarítmica, $Q^{2}(10)$ corresponde a Q-estatística de Ljung-Box para o décimo lag do erro quadrático, ARCH (10) refere-se ao Teste ARCH-LM para o lag 10,

Tabela 5. Parâmetros do modelo RiskMetrics.

\begin{tabular}{ll}
\hline & \multicolumn{1}{c}{ Normal } \\
\hline Constante da variância & $0,00098(0,0371)$ \\
ARCH (Alpha) & 0,05000 \\
GARCH (Beta) & 0,95 \\
& \\
Log-verossimilhança & 4980,306 \\
AIC & $-5,209021$ \\
Q $^{2}(10)$ & 6,26523 \\
ARCH (10) & 1,349 \\
\hline
\end{tabular}

Os valores entre parênteses correspondem ao p-valor de interpretação direta, Log-verossimilhança é o valor máximo da função de verossimilhança logarítmica, $\mathrm{Q}^{2}(10)$ corresponde a Q-estatística de Ljung-Box para o décimo lag do erro quadrático, $\mathrm{ARCH}$ (10) refere-se ao Teste ARCH-LM para o lag 10,

ao nível de $1 \%$ pela estatística F do teste ARCH-LM com 10 lags.

O efeito memória longa na volatilidade também pode ser observado no retorno do Ibovespa, por apresentar um valor significativo do parâmetro fracionário $d$ dos modelos FIGARCH e HYGARCH para todas as distribuições testadas. Esse efeito já havia sido testado e comprovado, conforme apresentado por Gaio e Sáfadi (2008). A presença de memória longa na volatilidade sugere uma evidência empírica de que a os retornos do Ibovespa apresentam uma baixa à média reversão por processo fracionalmente integrado.

Pelo Critério de Informação de Akaike (AIC), apresentado nas Tabelas 2-5, observa-se que o modelo FIGARCH $(1, \mathrm{~d}, 1)$ com distribuição t-Student Assimétrica obteve o melhor desempenho de ajustamento por apresentar o menor valor de AIC, comparado com os demais. Essa afirmação aponta para duas vertentes distintas; a primeira, que derruba a hipótese de que os retornos do Ibovespa se comportam conforme uma distribuição normal; e, a segunda, a qual demonstra que o modelo RiskMetrics apresentado pelo banco norte americano J.P. Morgan não se adequa à realidade do mercado acionário brasileiro, como uma ferramenta de estimação da volatilidade.

A supremacia do modelo FIGARCH $(1, \mathrm{~d} 1)$ para estimar a volatilidade de retornos em mercados acionários também foi observada em outros estudos, como foi o caso do Wu e Shieh (2007), aplicados aos retornos futuros do T-Bond e Tang e Shieh (2006), que investigaram a partir dos índices de mercado futuro S\&P500, Nasdaq100 e Dow Jones.

A Tabela 6 apresenta os p-valores referentes à estatística de falha do teste LR de Kupiec para a estimação do Value-at-Risk, calculados dentro da amostra, por meio dos modelos $\operatorname{GARCH}(1,1)$, FIGARCH $(1, \mathrm{~d}, 1), \mathrm{HYGARCH}(1, \mathrm{~d}, 1)$ e Riskmetrics para posições curtas e longas, assumindo diferentes distribuições para os retornos.

Os resultados da Tabela 6 evidenciam que os modelos ajustados com a distribuição $t$-Student Assimétrica possuem melhor desempenho, comparados com as distribuições Normal e $t$-Student, tanto para posições curtas e longas. Conforme expresso na seção 2.4, o percentual de retornos fora da fronteira VaR deve ser igual ao intervalo de confiança testado, ou seja, para um $\alpha=0,95$ ou $95 \%$, espera-se que apenas 5\% dos retornos não sejam captados pelo modelo VaR. Se isso não ocorrer, diz-se que este modelo não possui boa qualidade preditiva. A hipótese de nulidade do teste é dada quando o $\alpha$ for igual à razão de falha.

Por conseguinte, para todos os casos, aceita-se a hipótese nula para a distribuição $t$-Student Assimétrica, 
Tabela 6. Taxa de razão de falha calculada dentro da amostra para o Ibovespa.

\begin{tabular}{|c|c|c|c|c|c|c|}
\hline & \multicolumn{3}{|c|}{ VaR para posições curtas } & \multicolumn{3}{|c|}{ VaR para posições longas } \\
\hline & $95 \%$ & $97,5 \%$ & $99 \%$ & $5 \%$ & $2,5 \%$ & $1 \%$ \\
\hline \multicolumn{7}{|l|}{$\operatorname{GARCH}(1,1)$} \\
\hline Normal & 0,010 & 0,009 & 0,009 & 0,111 & 0,032 & 0,004 \\
\hline$t$-Student & 0,014 & 0,000 & 0,010 & 0,017 & 0,033 & 0,087 \\
\hline$t$-Student assimétrica & 0,716 & 0,392 & 0,080 & 0,496 & 0,447 & 0,835 \\
\hline \multicolumn{7}{|l|}{$\operatorname{FIGARCH}(1, d, 1)$} \\
\hline Normal & 0,059 & 0,050 & 0,137 & 0,038 & 0,145 & 0,034 \\
\hline$t$-Student & 0,007 & 0,001 & 0,009 & 0,029 & 0,062 & 0,194 \\
\hline t-Student assimétrica & 0,428 & 0,102 & 0,219 & 0,376 & 0,298 & 0,624 \\
\hline \multicolumn{7}{|l|}{$\operatorname{HYGARCH}(1, d, l)$} \\
\hline Normal & 0,005 & 0,005 & 0,080 & 0,037 & 0,045 & 0,002 \\
\hline t-Student & 0,007 & 0,000 & 0,010 & 0,029 & 0,033 & 0,194 \\
\hline$t$-Student assimétrica & 0,428 & 0,102 & 0,219 & 0,376 & 0,298 & 0,624 \\
\hline \multicolumn{7}{|l|}{ RiskMetrics } \\
\hline Normal & 0,493 & 0,050 & 0,465 & 0,010 & 0,000 & 0,000 \\
\hline
\end{tabular}

comprovando seu bom desempenho na predição do VaR no mercado acionário brasileiro.

A evidência de superioridade da distribuição t-Student para o cálculo do VaR também pode ser verifica em diferentes mercados mundiais, conforme os estudos de Giot e Laurent (2004) para os índices CAC40 e SP500, Tang e Shieh (2006), aplicados aos S\&P500, Nasdaq e Dow Jones, Wu e Shieh (2007), para o T-Bond e Giot e Laurent (2003a), aplicados a commodities agrícolas.

Comparando a capacidade do Riskmetrics aos modelos de variância condicional, percebe-se claramente a ineficiência do modelo da J. P. Morgan em estimar o risco de mercado na realidade brasileira. Para o cálculo das posições longas, é rejeitada a hipótese nula para os três intervalos 5\%,2,5\% e $1 \%$ do modelo Riskmetrcs, ao nível de 5\% de confiança. Já para as posições curtas, o Riskmetrics é rejeitado, ao nível de $10 \%$, no intervalo de $97,5 \%$. Isso aponta que o pacote disponibilizado pelo J. P. Morgan possui baixa validade para ser utilizado como ferramenta única de controle de risco no mercado acionário brasileiro. Porém esse pacote é um dos mais utilizados pelos profissionais de gestão de risco, devido a seu fácil manuseio e rapidez na predição.

Entre os modelos de variância condicional, não se pode afirmar qual obteve o melhor desempenho na predição do VaR diário, uma vez que os p-valores do teste de Kupiec (1995) estão na área de aceitação da hipótese nula para todos os modelos em todo os intervalos de previsão, com a utilização da distribuição de $t$-Student Assimétrica, tanto para posições curtas quanto longas.
Porém, quando se analisa a distribuição normal em específico, o modelo FIGARCH $(1, \mathrm{~d}, 1)$ possui melhor desempenho preditivo do VaR comparado com os outros. Todos os p-valores estão na área de aceitação da hipótese nula, ao passo que, para o modelo GARCH $(1,1)$, seus p-valores só aceitam $\mathrm{H}_{0}$ nos intervalos de $5 \%$ e $2,5 \%$ para posições longas e, no modelo HYGARCH $(1, \mathrm{~d}, 1)$, somente nos intervalos $99 \%$ (posição curta), $5 \%$ e $2,5 \%$ (posições longas) aceita-se a hipótese nula do teste de Kupiec (1995). Essa evidência expressa a existência de longa dependência nas volatilidades dos retornos do Ibovespa, que podem ser captados facilmente pelos modelos FIGARCH $(1, \mathrm{~d} 1)$ e HYGARCH $(1, \mathrm{~d}, 1)$.

A real contribuição do VaR está relacionada à habilidade de se prever as possíveis quedas ou altas do preço das ações do mercado de capital, para que seus investidores e gestores de carteira possam obter maior controle sobre o capital apurado nas transações, evitando perdas bruscas.

A Tabela 7, apresenta os valores referentes à estatística de falha do teste LR de Kupiec, aqui representada pelo p-valor de interpretação direta, para a previsão do Value-at-Risk calculado um passo a frente $(t+1)$, fora da amostra, por meio dos modelos GARCH $(1,1)$, FIGARCH $(1, d, 1)$, HYGARCH $(1, \mathrm{~d}, 1)$ e Riskmetrics para posições curtas e longas, assumindo diferentes distribuições para os retornos.

Para o teste de Kupiec (1995), foi realizada uma previsão fora da amostra com 500 possíveis valores de retornos diários. A utilização desse tamanho de amostra teve como referência os trabalhos de Tang e Shieh (2006) e Wu e Shieh (2007). 
Tabela 7. Taxa de razão de falha calculada fora da amostra para o Ibovespa.

\begin{tabular}{lcccccc}
\hline & \multicolumn{3}{c}{ VaR para posições curtas } & \multicolumn{3}{c}{ VaR para posições longas } \\
\cline { 2 - 7 } & $\mathbf{9 5 \%}$ & $\mathbf{9 7 , 5 \%}$ & $\mathbf{9 9 \%}$ & $\mathbf{5 \%}$ & $\mathbf{2 , 5 \%}$ & $\mathbf{1 \%}$ \\
\hline GARCH $(1,1)$ & & & & & & \\
Normal & 0,304 & 0,122 & 0,431 & 0,351 & 0,660 & 1,000 \\
$t$-Student & 0,502 & 0,122 & 0,431 & 0,038 & 0,392 & 1,000 \\
$t$-Student assimétrica & 0,502 & 1,000 & 1,000 & 0,351 & 0,660 & 1,000 \\
& & & & & & \\
FIGARCH $(1, d, 1)$ & & & & & & \\
Normal & 0,305 & 0,123 & 0,432 & 0,220 & 0,393 & 0,432 \\
$t$-Student & 0,305 & 0,123 & 0,432 & 0,072 & 0,393 & 0,432 \\
$t$-Student assimétrica & 0,742 & 0,639 & 1,000 & 1,000 & 0,660 & 0,432 \\
& & & & & & \\
HYGARCH $(1, d, 1)$ & & & & & & \\
Normal & 0,315 & 0,122 & 0,432 & 0,220 & 0,393 & 0,432 \\
$t$-Student & 0,502 & 0,123 & 0,432 & 0,072 & 0,393 & 0,432 \\
$t$-Student Assimétrica & 0,742 & 1,000 & 1,000 & 1,000 & 0,660 & 0,432 \\
& & & & & & \\
RiskMetrics & 0,000 & 0,000 & 0,000 & 0,000 & 0,000 & 0,000 \\
Normal & & &
\end{tabular}

Nota: os valores correspondem ao p-valor de interpretação direta, cuja hipótese nula é de que $f=\alpha$,

Os resultados apresentados na análise fora da amostra (Tabela 7) não se diferenciam muito das análises dentro da amostra (Tabela 6), o que evidencia a supremacia dos modelos de variância condicional - GARCH $(1,1)$, FIGARCH $(1, \mathrm{~d}, 1)$ e HYGARCH $(1, \mathrm{~d}, 1)$ - para todas as estimativas do VaR. Sua estatística de teste, em todos os casos, está na área de aceitação da hipótese nula ao nível de confiança de $10 \%$.

A metodologia Riskmetrics do J.P. Morgan obteve um desempenho ainda pior para o teste Kupiec (1995) calculados fora da amostra. A taxa de razão de falha, em todos os intervalos e posições, apresentou-se totalmente diferente do esperado. Para todos os casos, o p-valor foi igual a 0,00 , levando a estatística de teste para a área de rejeição de $\mathrm{H}_{0}$. Isso mostra que esta metodologia superestima os valores reais de retorno.

\section{Considerações finais}

O presente estudo propôs uma análise comparativa de alguns métodos de cálculo do Value-at-Risk (VaR) para o mercado acionário brasileiro, levando em consideração a presença de longa dependência em seus retornos (memória longa). Pretendeu-se fazer uma comparação entre modelos de extração da volatilidade para o cálculo do VaR, comparados com o modelo bastante utilizado pelos gestores de risco desenvolvido pelo J. P. Morgan e disponibilizado gratuitamente a qualquer instituição financeira, denominado Riskmetrics.

Os resultados obtidos sugerem que o pacote desenvolvido pelo J. P. Morgan não se aplica adequadamente à realidade do mercado acionário brasileiro, como ferramenta de gestão e controle do risco das oscilações dos preços das ações de empresas negociadas na Bolsa de Valores de São Paulo.

Entretanto, os modelos que consideram o efeito de memória longa na volatilidade condicional dos retornos do Ibovespa, em especial o modelo FIGARCH $(1, \mathrm{~d}, 1)$, foram os que obtiveram melhor ajuste e desempenho preditivo do risco de mercado (Value-at-Risk), conforme valores apresentados pelo teste de razão de falha proposto por Kupiec (1995).

A principal contribuição deste trabalho centra-se no aspecto metodológico para as futuras previsões de $\mathrm{VaR}$ a qualquer período de interesse. No entanto, essa medida de risco apresenta bons resultados quando aplicada a um mercado com baixas inversões de comportamento. Para épocas de grandes instabilidades e alta volatilidade, o VaR tende a subestimar estes valores e, portanto, seria necessária alguma outra medida de risco, de forma a complementar tal análise.

Pretende-se, em trabalhos futuros, a ampliação deste estudo, levando em conta uma análise de caráter multivariado; em outras palavras, seria a aplicação do VaR em carteiras de investimento e não somente a um comportamento de preços únicos. Para isso, serão necessários estudos que comparem diferentes métodos de estimação da covariância entre os portfólios, utilizando, como proxy, o modelos de volatilidade multivariados, como é o caso dos modelos DCC, GARCH BECK e CC. Sugere-se, também, um estudo mais amplo incorporando não somente uma análise comparativa com modelos de volatilidade 
condicional, mas também, englobando as Teorias de Valores Extremos e teste de stress.

\section{Referências}

BAILLIE, R. T.; BOLLERSLEV, T.; MIKKELSEN, H. O. Fractionally integrated generalized autoregressive conditional heteroskedasticity. Journal of Econometrics, v. 74, n. 1, p. 3-30, 1996. http://dx.doi.org/10.1016/ S0304-4076(95)01749-6

BERA, A. K.; JARQUE, C. M. Efficient tests for normality, homoscedasticity and serial independence of regression redisuals: Monte Carlo evidence. Economic Letters 7, v. 4, p. 313-318, 1981

BEST, P. Implementing Value-at-Risk. New York: John Wiley \& Sons, 1998. http://dx.doi. org/10.1002/0470013303

BOLLERSLEV, T. Generalized autoregressive conditional heteroskedasticity. Journal of Econometrics, v. 31, p. 307-327, 1986. http://dx.doi. org/10.1016/0304-4076(86)90063-1

DAVIDSON, L. Moment and memory properties of linear conditional heteroscedasticity models. Journal of Business and Economics Statistics, v. 22, p. 16-29, 2004. http://dx.doi.org/10.1198/073500103288619359

DICKEY, D. A.; FULLER, W. A. Distribution of the Estimators for Autoregressive Time Series with a Unit Root. Journal of the American Statistical Association, v. 74, p. 427-431, 1979.

DOORNIK, J. A. An Object Oriented Matrix Programming Language. 4th ed. Timberlake Consultant Ltd., 2001.

DOWD, K. Beyond Value at Risk. John Wiley \& Sons, 1998.

ENGLE, R. F. Autoregressive conditional heteroscedasticity with estimates of the variance of United Kingdom inflation. Econometrica, v. 50, n. 4, p. 987-1007, 1982. http://dx.doi.org/10.2307/1912773

ENGLE, R. F.; BOLLERSLEV, T. Modelling the persistence of conditional variances. Econometric Reviews, v. 5, n. 1, p. 1-50, 1986. http://dx.doi. org/10.1080/07474938608800095

GAIO, L. E.; SAFADI, T. Memória longa na volatilidade do índice Bovespa: Uma análise utilizando modelos da classe ARCH. Revista de Economia e Administração, v. 7, n. 2, p. 228-243, 2008.

GALDI, F. C.; PEREIRA, L, M. Valor em Risco (VaR) utilizando modelos de previsão de volatilidade: EWMA, GARCH e Volatilidade Estocástica. Brazilian Business Review, v. 4, n. 1, p. 74-95, 2007.

GIOT, P.; LAURENT, S. Market risk in commodity markets: a VaR approach. Energy Economics, v. 25, p. 435-457, 2003a. http://dx.doi.org/10.1016/ S0140-9883(03)00052-5

GIOT, P.; LAURENT, S. Value-at-Risk for long and short trading positions. Journal of Applied Econometrics, v. 18, p. 641-664, 2003b. http://dx.doi.org/10.1002/ jae. 710

GIOT, P.; LAURENT, S. Modelling daily Value-at-Risk using realizes volatility and ARCH type models. Journal of Empirical Finance, v. 11, p. 379-398, 2004. http:// dx.doi.org/10.1016/j.jempfin.2003.04.003

GOULART, C. P. et al. Previsão da volatilidade no mercado interbancário de câmbio. RAE - Revista de
Administração de Empresas, v. 45, p. 86-101, 2005. Edição especial Minas Gerais.

GRANGER, C. W. J. Long memory relationships and the aggregation of dynamic models. Journal of Econometrics, v. 14, p. 8-227, 1980.

HOSKING, J. R. M. Fractional differencing. Biometrica, v. 68, p. $76-165,1981$.

HUANG, Y. C.; LIN, B. J. Value-ar-Risk Analysis for Taiwan Sotck Index Futures: Fat Tails and Conditional Asymmetries in return innovations. Review of Quantitative Finance and Accounting, v. 22, p. 79-95, 2004. http://dx.doi.org/10.1023/ B:REQU.0000015851.78720.a9

JORION, P. Value-at-Risk: The new benchmark for controlling market risk. New York: MacGraw-Hill, 1997.

KUPIEC, P. H. Techiques for verifying the accuracy of risk measurement models. The Journal of Derivatives, v. 3, n. 2, p. 73-84, 1995. http://dx.doi.org/10.3905/ jod.1995.407942

KWIATKOWSK, D. et al. Testing the null hypothesis of stationary against the alternative of a unit root. Journal of Econometrics, v. 54, p. 159-178, 1992. http://dx.doi. org/10.1016/0304-4076(92)90104-Y

LAURENT, S.; PETERS, J. P. G@RCH 2,3: An Ox pachage for estimationg and forecasting various $\mathrm{ARCH}$ models. Journal of Economic Surveys, v. 16, p. 447-485, 2002. http://dx.doi.org/10.1111/1467-6419.00174

MACIEL, L. S. Valor em risco de longo prazo: uma abordagem para modelos da Família Arch e redes neuronais. Revista Economia e Gestão, v. 10, n. 24, p. 103-125, 2010.

MOL, A. L. R. Value at Risk como medida de risco da volatilidade dos ajustes diários em mercados futuros de café. 2003. 102 f. Dissertação (Mestrado em Administração)-Universidade Federal de Lavras, Lavras, 2003.

MOREIRA, J. M. S.; LEMGRUBER, E. F. O uso de dados de alta frequencia na estimação da volatilidade e do valor em risco para o Ibovespa. Revista Brasileira de Economia, v. 58, n. 1, p. 100-120, 2004. http://dx.doi. org/10.1590/S0034-71402004000100005

MORETTIN, P. A.; TOLOI, C. M. C. Análise de séries temporais. São Paulo: Edgard Blücher, 2004.

MORGAN, J. P. RiskMetrics. 4th ed. New York, 1996. Technical document.

MOTA, B.; FERNANDES, M. Desempenho de estimadores de volatilidade na bolsa de valores de São Paulo. Revista Brasileira de Economia, v. 58, n. 3, p. 429-448, 2004. http://dx.doi.org/10.1590/S0034-71402004000300006

NIIGUEZ, T. M. Volatility and Var forecasting in the Madrid Stock Exchange. Spanish Economic Review, v. 10, p. 169-196, 2008. http://dx.doi.org/10.1007/ s10108-007-9030-6

PHILLIPS, P. C. B.; PERRON, P. Testing for a unit root in time series regression. Biometrika, v. 75, p. 335-346, 1988. http://dx.doi.org/10.1093/ biomet/75.2.335

SILVA, W. S. Modelagem da volatilidade dos índices financeiros IBOVESPA, Dow Jones e Standard \& Poors utilizando modelos da classe ARCH. 2003. $91 \mathrm{f}$. Dissertação (Mestrado em Estatística e Experimentação Agropecuária)-Universidade Federal de Lavras, Lavras, 2003. 
SO, M. K. P.; YU, P. L. H. Empirical analysis of GARCH models in value at risk estimation. Journal of International Financial Markets, Institutions and Money, v. 16, n. 2, p. 180-197, 2006. http://dx.doi. org/10.1016/j.intfin.2005.02.001

TANG, T. L.; SHIEH, S. J. Long memory in stock index futures markets: A value at risk approach. Physica A, v. 366, p. 437-448, 2006. http://dx.doi.org/10.1016/j. physa.2005.10.017

WU, P. T.; SHIEH, S. J. Value-at-Risk analysis for longterm interest rate futures: Fat-tail and long memory in return innovations. Journal of Empirical Finance, v. 14, p. 248-259, 2007. http://dx.doi.org/10.1016/j. jempfin.2006.02.001 